\title{
Spatially and Temporally Optimized Video Stabilization
}

\author{
Yu-Shuen Wang, Feng Liu, Member, IEEE, Pu-Sheng Hsu, and Tong-Yee Lee, Senior Member, IEEE
}

\begin{abstract}
Properly handling parallax is important for video stabilization. Existing methods that achieve the aim require either $3 \mathrm{D}$ reconstruction or long feature trajectories to enforce the subspace or epipolar geometry constraints. In this paper, we present a robust and efficient technique that works on general videos. It achieves high-quality camera motion on videos where 3D reconstruction is difficult or long feature trajectories are not available. We represent each trajectory as a Bézier curve and maintain the spatial relations between trajectories by preserving the original offsets of neighboring curves. Our technique formulates stabilization as a spatialtemporal optimization problem that finds smooth feature trajectories and avoids visual distortion. The Bézier representation enables strong smoothness of each feature trajectory and reduces the number of variables in the optimization problem. We also stabilize videos in a streaming fashion to achieve scalability. The experiments show that our technique achieves high-quality camera motion on a variety of challenging videos that are difficult for existing methods.
\end{abstract}

Index Terms-Video stabilization, warping, optimization, Bézier curve

\section{INTRODUCTION}

$\mathrm{M}$ OST amateur videos are captured using hand-held cameras. They are often very shaky and difficult to watch. Therefore, video stabilization techniques have been developed to smooth shaky camera motion. Most existing methods work in 2D and are efficient and robust. However, these methods use homography, which cannot model parallax induced by a moving camera imaging a 3D scene, so they cannot aggressively stabilize shaky camera motions.

To handle parallax well, 3D video stabilization methods apply 3D reconstruction from structure from motion (SFM) [1] or depth acquisition from sensors [2]. However, SFM solves a highly nonlinear optimization problem to reconstruct a 3D scene, which is computationally expensive and is often brittle. Depth acquisition is limited to depth cameras and thus is not applicable to general videos. Closely related to our technique, where scene modeling is not required, Liu et al. [3] developed a method that makes use of "eigen-trajectories," a middle-level representation between $2 \mathrm{D}$ and $3 \mathrm{D}$, and smoothes camera motion in the subspace of feature trajectories. However, the method requires a certain number of long feature trajectories. It potentially breaks down when such trajectories are not enough, which usually occurs in videos suffering from serious blurriness. Goldstein and Fattal developed a

- Y.-S. Wang is with the Department of Computer Science, National Chiao-Tung University, Room EC714, 1001 University Road, Hsinchu 300, Taiwan, R.O.C. E-mail: yushuen@cs.nctu.edu.tw.

- F. Liu is with the Department of Computer Science, Portland State University, PO Box 751 - CMPS, Portland, OR 97207-0751.

E-mail: fliu@cs.pdx.edu.

- P.-S Hsu and T.-Y. Lee are with the Department of Computer Science and Information Engineering, National Cheng-Kung University, No. 1, University Road, Tainan 701, Taiwan, R.O.C.

E-mail: tonylee@mail.ncku.edu.tw, prindrock@gmail.com.

Manuscript received 14 Aug. 2012; revised 3 Dec. 2012; accepted 31 Dec. 2012; published online 23 Jan. 2013.

Recommended for acceptance by M. Agrawala.

For information on obtaining reprints of this article, please send e-mail to: tvcg@computer.org, and reference IEEECS Log Number TVCG-2012-08-0159. Digital Object Identifier no. 10.1109/TVCG.2013.11. method that uses a projective scene reconstruction [4] to handle parallax without 3D reconstruction. Their method, however, cannot work well on videos with strong occlusions and non-Lambertain surfaces.

In this paper, we introduce a video stabilization method that can handle parallax and is robust to poor motion estimation results. Our key insight is that the tracked features are spatially and temporally correlated. Solving a video stabilization problem in separate passes, say, computing spatial relations (such as planar assumption, SFM, and subspace extraction) followed by smoothing the reduced parameters, cannot make use of all the feature trajectories. Accordingly, we formulate stabilization as a spatial-temporal optimization problem that considers both smoothing and spatial rigidity simultaneously to handle parallax. This formulation enables our system to utilize both long and short feature trajectories and approach global optimal solutions when stabilizing videos. To achieve high performance, we represent each smoothed feature trajectory using a Bézier curve when minimizing the objective function. Since the unknowns are reduced from feature positions to only curve control points, heavy computational cost and memory consumption can be prevented. By setting a lower degree to Bézier curves, this representation also achieves strong stabilization because each smoothed feature trajectory is interpolated from the optimized control points. Although the Bézier curve idea may be not new [3], [5], [6], [7], we embed this reduced model in the spatial-temporal optimization problem and enable high-performance video stabilization that creates high-quality camera motion.

During the optimization, we explicitly constrain the spatial offsets of neighboring features in each frame when smoothing their motions. Each local region is enforced to undergo a rigid transformation because the stabilization will typically not change the viewpoint too much and thus will not distort the interior content, unless the video suffers from rolling shutter artifacts. In addition, the Bézier representation enables our method to directly smooth feature trajectories and thus, our system can handle parallax 
well. Although the number of variables in each trajectory has already reduced, the computational cost of our system would still increase as more and more trajectories are added when the video becomes long. We, therefore, optimize the feature trajectories within the moving time window in a streaming manner while constraining the smoothness in the overlapping frames to achieve scalability. Once all trajectories are optimized, we consider the smoothed features as positional constraints and apply the content preserving methods to warp video frames sequentially. Finally, a cropping window is determined to capture the maximal area containing only valid information to produce the stabilizing result.

We demonstrate the effectiveness of our technique by comparing it to state-of-the-art techniques [3], [4], [8] on a variety of videos containing large parallax and strong occlusions. Experiments show that our method can handle parallax and is robust to challenging examples. Since the L1-Optimization method provided by Youtube [8] is the most robust technique among these recent works, we also evaluate the performance of our framework by conducting a user study with 74 participants, which showed strong preference of our method over [8].

\section{Related Work}

Video stabilization techniques have been developed to smooth shaky camera motions. Two-dimensional stabilization methods estimate a homography sequence from the input video, dampen this sequence, and warp video frames based on the smoothed sequence [9], [10], [11], [12], [13], [14]. A recent method achieves aggressive smoothing using L1 optimization [8], in which the gradient of the camera motion at each frame can be strongly suppressed, to produce very high-quality results. However, the use of 2D motion models still limits its ability to handle parallax.

Three-dimensional stabilization methods use SFM to estimate 3D camera motion and scene structure, followed by smoothing the camera motion. These methods then render a new video according to the smoothed camera path using image-based rendering [15], [16], homography approximation [17], or content-preserving warp [1], [18]. However, SFM is a difficult and time-consuming computer vision problem. It often fails when the video 1) lacks parallax, 2) contains camera zooming, or 3) suffers from rolling shutter artifacts. Therefore, these $3 \mathrm{D}$ methods are not practical in many examples.

Liu et al.'s method [3] smoothes feature trajectories with subspace constraints to achieve robustness and efficiency. The tracked feature trajectories are factorized into lowdimensional key bases and reconstruction coefficients. Their method then smoothes the bases followed by trajectory reconstruction to stabilize videos. However, the streaming factorization requires long feature trajectories to cover the entire factorization window, which do not usually appear in videos containing dynamic backgrounds and large foreground motions. Goldstein and Fattal [4] applied the projective reconstruction to account for simple geometric relations between points and epipolar lines, which raises the robustness of scene modeling. They also derived a time-view point reprojection to stabilize trajectories of moving objects so that the defined warping constraints can be spread more uniformly across the frame to capture the scene shape. However, the projective reconstruction relies on relative positions of corresponding features between images, so the method fails at examples that have strong occlusions and non-Lambertian surfaces. Liu et al. [2] stabilized videos that are captured by depth cameras, in which the relative motions of neighboring content can be estimated and then preserved when smoothing the camera motion. As this method requires depth acquisition, it cannot work for regular videos. In contrast, our method, which smoothes each feature motion while retaining the offsets to neighboring features in the spatial-temporal coordinate system, achieves high-quality stabilization without requiring long feature trajectories, scene reconstruction, or depth acquisition.

Although CMOS technique is becoming appealing due to its low-cost and low-power consumption, its row by row readout causes rolling shutter artifacts. To remove the artifacts, methods such as [19], [20] estimate the parametric motion between consecutive frames and apply the regularization method to minimize temporal derivative of the motion. Recently, Grundmann et al. [21] introduced a calibration-free algorithm, which removes blind rolling shutters using mixture model of homographies, to handle challenging artifacts introduced by various CMOS cameras.

\section{Feature Trajectory Smoothing}

We stabilize videos by smoothing the trajectories that are integrated from the KLT features [22]. Similar to the subspace method [3], the tracked features on moving objects are ruled out in advance using the epipolar constraint [23]. We then smooth the remaining feature trajectories while retaining their spatial relations to stabilize videos. Note that this outlier rejection is not necessarily perfect because the remaining foreground features have nearly static motions and those regions can be treated as backgrounds.

Let the $i$ th trajectory be $\mathbf{P}_{i}=\left\{\mathbf{p}_{i}^{m}, \mathbf{p}_{i}^{m+1}, \ldots, \mathbf{p}_{i}^{n}\right\}$, where $\mathbf{p}_{i}=\left(x_{i}, y_{i}\right) \in \mathcal{R}^{2}$ is the feature position, and $m$ and $n$ are the start and the end frames of $\mathbf{P}_{i}$, respectively. Our goal is to solve an optimization problem that can minimize the acceleration of $\mathbf{P}_{i}$ in each frame while constraining the offsets of neighboring trajectories to be consistent within the input video. To achieve high performance, we represent each smoothed trajectory using a Bézier curve and reduce the unknown variables from all feature positions to curve control points. This reduced model also achieves strong stabilization because the smoothed feature positions are interpolated from the control points. We show the details of our technique in the following sections.

\subsection{Objective Function}

Spatial rigidity preservation. As proved in the work of [3], individual smoothing of feature trajectories distorts geometric relationships of video objects. Therefore, we retain the spatial rigidity when stabilizing a video to preserve neighboring feature trajectories to have similar treatments. Specifically, we compute the neighbor relations between features in each frame using the Delaunay triangulation and enforce each triangle to undergo a rigid transformation. 
This constraint works well in most videos that are free from rolling shutter artifacts because the change to the camera motion is typically small in video stabilization. That is, triangles are allowed to move and rotate but their sizes and shapes should be retained. Since features are not uniformly distributed, they may be very different in consecutive frames (see Fig. 2) and thus, preserving only relative positions of features is not sufficient to stabilize the regions that have no features in the current frame but have some previously. We thus add four corners of each frame when computing the Delaunay triangulation and introduce the energy term

$$
\Omega_{c}=\sum_{t} \sum_{f} \sum_{\{i, j\} \in \mathcal{E}(f)}\left|\left(\mathbf{r}_{i}^{\prime t}-\mathbf{r}_{j}^{\prime t}\right)-\mathbf{R}^{t}\left(\mathbf{r}_{i}^{t}-\mathbf{r}_{j}^{t}\right)\right|_{F}^{2},
$$

where $t$ is the frame index, $\mathcal{E}(f)$ denotes the edges of triangle $f, \mathbf{r}=\{\mathbf{c}, \mathbf{p}\}, \mathbf{c}$ is the original corner position, $\mathbf{R}$ is the unknown rotation matrix, and $\mathbf{r}^{\prime}$ is the smoothed version of $\mathbf{r}$.

Original camera motion approximation. Clearly, video stabilization cannot deviate the smoothed camera motion from its original trajectory too much because the motion usually has important meanings. This requirement also reduces the content loss because video frames are transformed during the stabilization and only the overlapped areas throughout the entire video will remain. Therefore, let $\mathbf{p}^{\prime}$ be the smoothed feature position, we give the energy term:

$$
\Omega_{p}=\sum_{\mathbf{P}_{i}} \sum_{t}\left|\mathbf{p}_{i}^{\prime t}-\mathbf{p}_{i}^{t}\right|_{F}^{2} .
$$

Individual trajectory smoothing. To achieve video stabilization, we can minimize the acceleration of each feature motion using the energy term

$$
\Omega_{s}=\sum_{\mathbf{P}_{i}} \sum_{t}\left|\mathbf{p}_{i}^{\prime t-1}-2 \mathbf{p}_{i}^{\prime t}+\mathbf{p}_{i}^{\prime t+1}\right|_{F}^{2}
$$

However, minimizing the objective function $\Omega_{c}+\Omega_{p}+\Omega_{s}$ requires solving all feature positions in one step, where the number of unknown variables is tremendous. This requirement would lead to heavy computational cost and large memory consumption when a long and highresolution video is processed. In addition, as pointed out by Grundmann et al. [8], least-squares optimization satisfies (3), on average, which would result in small but nonzero gradients between consecutive frames. It means that the optimized video is only less shaky but still not stable (see Fig. 3 and our online supplemental video Bezier.mp4, which can be found on the Computer Society Digital Library at http://doi.ieeecomputersociety.org/ 10.1109/TVCG.2013.11). To solve these two problems, we represent the smoothed trajectory using a Bézier curve so that feature motions can be strongly smooth and the unknown variables are greatly reduced. Specifically, each node on a trajectory is written as $\mathbf{p}_{i}^{t}=\sum_{\ell=0}^{d} \omega_{i}^{t, \ell} \mathbf{q}_{i}^{\ell}$, where $\omega^{t, \ell}=\left(\begin{array}{l}d \\ \ell\end{array}\right)(1-r)^{d-\ell} r^{\ell}, d$ is the degree of Bézier curve, $\mathbf{q}_{i}$ are the control points and $r=\frac{t-n}{m-n}$ is the interpolation coefficient. Thus, our goal becomes approximating each jittering feature trajectory using a Bézier curve while retaining the offsets of neighboring features in each frame. Formally, we rewrite the unknown feature position $\mathbf{p}_{i}^{\prime t}$ in (1) and (2) to $\sum_{\ell=0}^{d} \omega_{i}^{t, \ell} \mathbf{q}_{i}^{\ell}$ and neglect (3) when solving the optimization.

Generally, a larger value of $d$ can retain more content after cropping while a smaller value enjoys stronger camera stabilization due to the different degrees of Bézier curve fitting. For generality, we set $d=2$ in all our experimental results, except the online supplemental video DegreeComparison.mp4, that we used to illustrate the effect of different degrees.

Reliability constraints. When video frames containing no features, there is no way to determine their smoothed positions. The minimization of (1) and (2) subject to the Bézeir constraint is not sufficient to stabilize such jittering videos because the unknowns are not correlated. Therefore, we slightly enforce the corners of these extremely difficult frames to have smooth transitions between consecutive time steps, so that our system can be robust to all challenging examples. Specifically, we add the energy term

$$
\Omega_{r}=\sum_{t \in \mathbf{U}} \sum_{i=0}^{3}\left|\mathbf{c}_{i}^{t-1^{\prime}}-2 \mathbf{c}_{i}^{t^{\prime}}+\mathbf{c}_{i}^{t+1^{\prime}}\right|_{F}^{2},
$$

where $i$ is the corner index and $\mathbf{U}$ is the set of frames that have no features.

\subsection{Optimization}

By integrating the mentioned energy terms, we search for the control points of Bézier curves that can minimize the objective function

$$
\Omega=\mathrm{w}_{c} \Omega_{c}+\mathrm{w}_{p} \Omega_{p}+\mathrm{w}_{r} \Omega_{r},
$$

to stabilize videos, where $\mathrm{w}_{c}, \mathrm{w}_{p}$, and $\mathrm{w}_{r}$ are the weighting factors $\left(\mathrm{w}_{c}=10, \mathrm{w}_{p}=1\right.$, and $\mathrm{w}_{r}=0.01$ in all our experiments). The optimization is solved by iteratively updating the unknown corners, the control points of Bézier curves and the rotation matrixes because they are correlated. Specifically, we first set $\mathbf{R}=\mathbf{I}$ and compute $\mathbf{q}$ and $\mathbf{c}$ by solving a linear system. $\mathbf{R}$ is then determined by first computing a homography transformation between the original and the smoothed features and corners, and followed by using the singular value decomposition to eliminate the shear and the scale components. Please refer to [24] for more optimization details. Once the control points are obtained, we compute the stabilized feature positions at each frame using $\hat{\mathbf{p}}_{i}^{t}=\sum_{\ell=0}^{d} \omega_{i}^{t, \ell} \mathbf{q}_{i}^{\ell}$.

It is known that the Bézier and the rigidity constraints conflict when handling videos that have parallax. Expecting each region to undergo an exact rigid transformation cannot stabilize the video since features with different depth values have different shaking magnitudes. When this conflict happens, our system can still smooth feature motions to handle parallax because our Bézier representation is a hard constraint but $\Omega_{c}$ is a soft one. While the triangles may be slightly distorted, each stabilized feature trajectory is definitely smooth because it is interpolated from the optimized Bézier control points. 


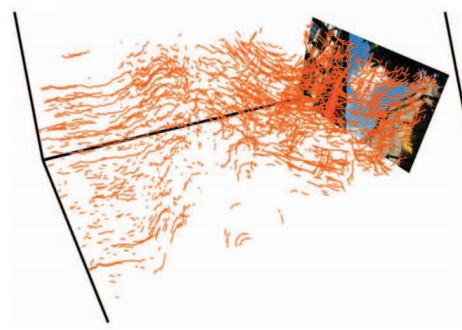

(a)

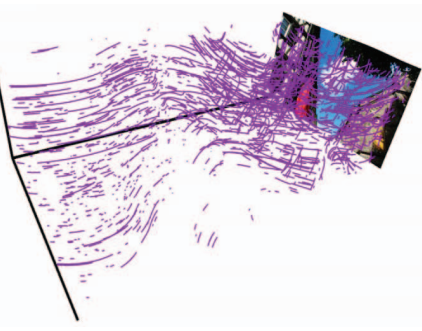

(b)

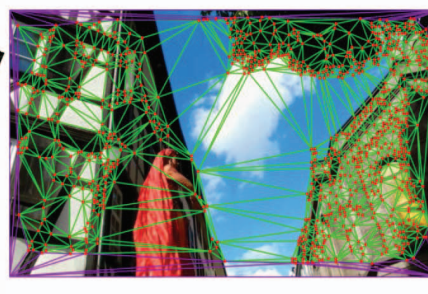

(c)

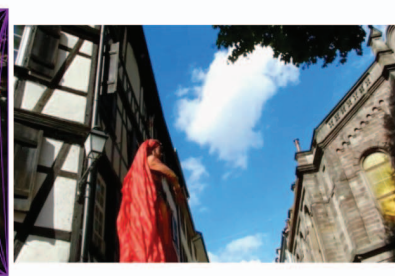

(d)

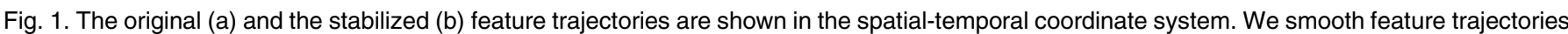

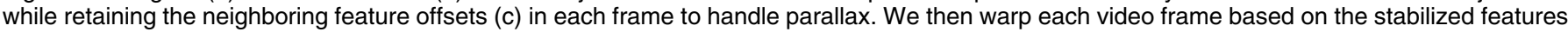
and crop the maximal overlapping area to produce the result (d).
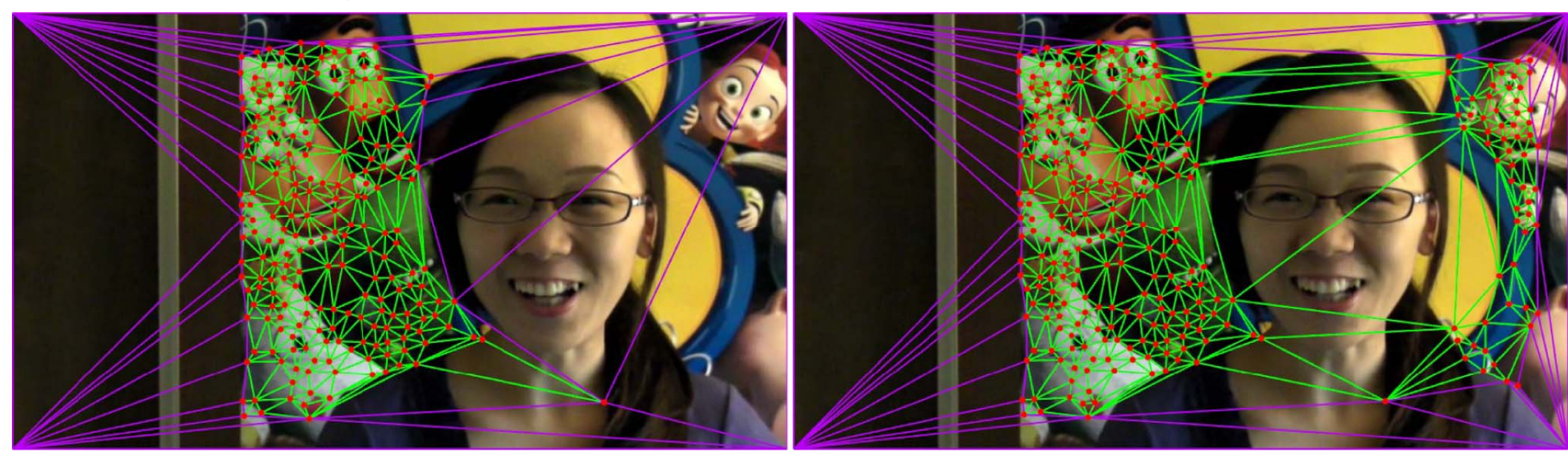

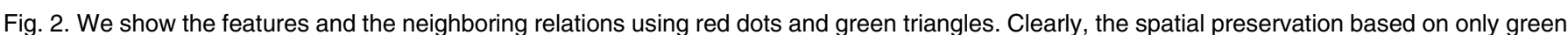

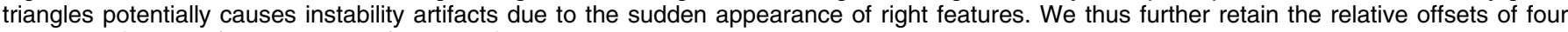
corners to features (purple triangles) in each frame to prevent this problem.

\subsection{Implementation Details}

Scalability. We solve for a subset of video frames instead of the whole video cube at a time to prevent the scalability problem, even though each feature trajectory is already transformed into a reduced model. This streaming strategy is very helpful to our system because the captured videos are usually long and the computational cost would increase rapidly. Therefore, we segment the video into shorter clips, with each clip containing $k$ frames, and with $g$ frames overlapping between consecutive clips $(k=200$ and $g=50$ in all our experiments). During the optimization of the $\lambda$ th clip, we solve for the smoothed feature positions from frame $k \times \lambda-g$ to frame $k \times(\lambda+1)$ (see Fig. 4). Hence, a long trajectory will be approximated using several Bézier curves. We thus set positional constraints in the overlapping frames to prevent the discontinuity artifacts caused by our streaming approach. Specifically,

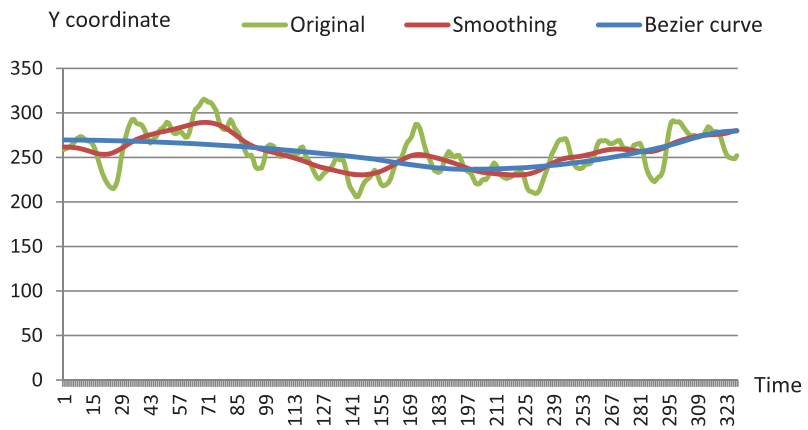

Fig. 3. We compare the smoothness of stabilized feature trajectories using acceleration minimization and Bézier curve fitting. when stabilizing the $\lambda^{t h}(\lambda>0)$ clip, we replace $\mathbf{p}_{i}^{t}$ by $\hat{\mathbf{p}}_{i}^{t}$ in (2) if $k \times \lambda-g \leq t \leq k \times \lambda$, where $\hat{\mathbf{p}}$ is the stabilized feature position obtained in the $(\lambda-1)$ th clip. Since some features are computed twice in consecutive clips, they may have very similar but different positions. We determine their smoothed positions by linear blending.

Bézier curve fitting. Apparently, a Bézier curve may not well fit the shape of a long and twisting trajectory. Although our streaming implementation that solves a subset of video frames at a time can ease the problem, it potentially happens to some complicated camera motions and results in waving artifacts. Therefore, before the minimization of $\Omega$ shown in (5), we compute a best fit Bézier curve for each trajectory without considering spatial rigidity. We then determine the fitting error by computing the largest distance between the pair of the original and the smoothed feature positions. For the trajectory having a fitting error larger than $\mu$ pixels, we partition it into two subtrajectories at that position. This process repeats until each partitioned trajectory can be well fitted. We set $\mu=\sqrt{\mathcal{A}}$ in our system, where $\mathcal{A}$ is the averaged triangle area.

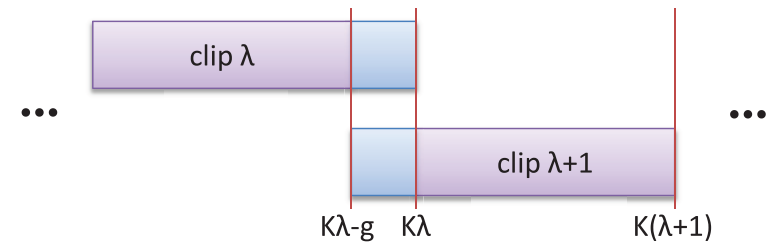

Fig. 4. We streamingly stabilize a long video to achieve scalability. The feature positions in the overlapping frames are smoothed to prevent the discontinuity artifacts. 


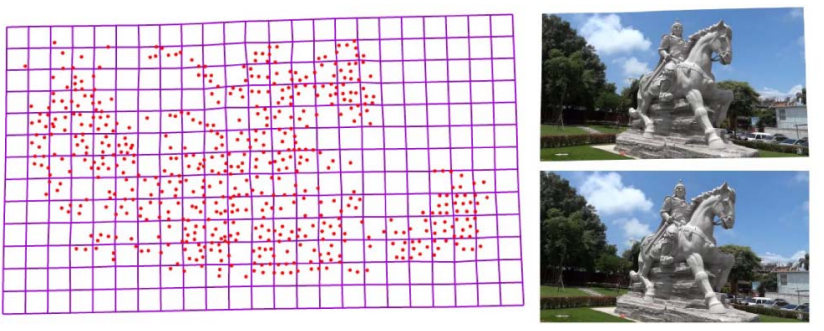

Fig. 5. (left) The warped quad mesh and the smoothed features. (top right) The warped frame. (bottom right) The original frame. We warp each video frame by enforcing features to be located at the smoothed positions so that some grid lines bend although each local region is transformed homographically.

\section{VIDEO WARPING}

We warp video frames according to the optimized feature positions to determine the result. We render each video frame based on the deformed grid mesh, rather than the triangular one shown in Fig. 2 because the appearing and the missing features are less reliable (pointed out by Liu et al. [1]), and additional stability constraints are needed.

Specifically, we represent each video frame using a regular grid mesh $\mathbf{M}^{t}=\left\{\mathbf{V}^{t}, \mathbf{E}^{t}\right\}$ with each quad covering roughly $10 \times 10$ pixels, where $\mathbf{v}_{i}^{t} \in \mathbf{V}^{t}$ and $\{i, j\} \in \mathbf{E}$ are the grid vertices and edges, respectively. During the frame warping, we expect each quad to undergo a homography transformation $\mathbf{H}^{t}$ while constraining each feature to locate at the smoothed position according to the feature reliability. The transformation $\mathbf{H}^{t}$ can be considered as a 2D stabilization and it is computed according to the original and the smoothed features and corners. We use this homography to stabilize the regions with no features, including homogeneous regions and moving foregrounds. Although the transformation $\mathbf{H}^{t}$ treats each moving foreground as a plane, our system does not introduce noticeable visual artifacts because the foreground motions are usually much larger than the jittering caused by depths.

Due to the discrete representation, we constrain each feature position using the linear combination of surrounding vertices when warping a video frame. We also compute the reliability value of each feature using $\gamma_{i}^{t}=$ $\min \left(1, \frac{t-m}{10}, \frac{n-t}{10}\right)$, which is mainly based on distances to end points of a trajectory. By considering the above criteria, we minimize the objective function $D_{h}^{t}+D_{p}^{t}$ to warp each video frame, where

$$
\begin{gathered}
D_{h}^{t}=\sum_{\{i, j\} \in \mathbf{E}^{t}}\left|\left(\hat{\mathbf{v}}_{i}^{t}-\hat{\mathbf{v}}_{j}^{t}\right)-\mathbf{H}^{t}\left(\mathbf{v}_{i}^{t}-\mathbf{v}_{j}^{t}\right)\right|_{F}^{2}, \\
D_{p}^{t}=\sum_{\hat{\mathbf{p}}_{i}^{t}} \gamma_{i}^{t}\left|\sum_{j \in \mathcal{L}_{i}^{t}} \delta_{j}^{t} \hat{\mathbf{v}}_{j}^{t}-\hat{\mathbf{p}}_{i}^{t}\right|_{F}^{2},
\end{gathered}
$$

$\mathcal{L}_{i}^{t}$ are the indexes of vertices surrounding $\hat{\mathbf{p}}_{i}^{t}$ and $\delta$ is the combination weight. Note that solving the mentioned objective function does not degenerate our algorithm to a 2D stabilization even though the energy term $D_{h}^{t}$ treats each video frame as a plane, because the features are still constrained at the smoothed positions by $D_{p}^{t}$. As Fig. 5 shows that the quad deformations are similar but not identical to ensure all features moving smoothly. Once all
TABLE 1

Video Information and the Corresponding Timing Statistics

\begin{tabular}{|c|c|c|c|}
\hline resolution & \# frame & \# trajectory & smoothing $(\mathrm{sec})$. \\
\hline $640 \times 360$ & 609 & 4580 & 6.685 \\
$720 \times 480$ & 389 & 15415 & 7.42 \\
$720 \times 480$ & 331 & 7016 & 4.368 \\
$1280 \times 720$ & 775 & 26921 & 22.72 \\
\hline
\end{tabular}

Note that the timing of $K L T$ feature extraction is not included

meshes are transformed to achieve stabilization, we linearly interpolate pixel colors in each quad and determine the maximal window that covers only valid information to produce the stabilized video.

\section{Results and Discussions}

We implemented and tested our algorithm on a desktop PC with Core i7 3.0-GHz CPU. We use the direct solver with Cholesky factorization to minimize $\Omega$ in (5) to achieve high precision. In general, the computational cost mainly depends on the number of feature trajectories. The trajectory lengths do not affect the performance too much because we have already transformed each trajectory into a Bézier curve and only control points are solved to obtain its smoothed version. In addition, we stabilize a subset of video frames sequentially instead of the whole video cube at a time to achieve scalability. The size of the linear system is thus small. For the example, we show in Fig. 1, there are 563,351 feature positions among 775 frames. Since we represent each trajectory using a quadratic Bézier curve, we solve for only $3 \times 7,008$ control points in average when stabilizing each 200 -frames segmented video clip so as to achieve high performance. On the other hand, we use the conjugate gradient method to warp each video frame because the frame can be prewarped using the homography transformation and used as an initial guess for fast convergence. We also leverage parallel implementation to speed up individual frame warping. Table 1 shows the timing statistics. Our method uses the Voodoo camera tracker [25] to track KLT features. While the KLT implementation used in our method is not real-time, feature trajectory estimation is a common step in existing video stabilization methods. Our method achieves real-time performance after we preprocess the input video to estimate the KLT feature trajectories.

We have tested diverse examples containing dynamic backgrounds, strong occlusions and large parallax to demonstrate the effectiveness of our system. All the results are generated automatically using the default parameters. Many of the examples involve large foreground objects occupying almost the whole video frame, making the stabilization rather challenging. While previous methods [3], [4], [8] all fail in such difficult examples, our technique successfully stabilizes the videos with no visual artifacts. To achieve a fair comparison, we also tested the videos chosen from previous methods to demonstrate that our system not only works well in our selected examples but also in theirs. Please refer to Figs. 1, 5, 6, and 7, the accompanying videos and our project webpage 1 for more results and comparisons,

1. http://people.cs.nctu.edu.tw/ yushuen/VideoStabilization/. 

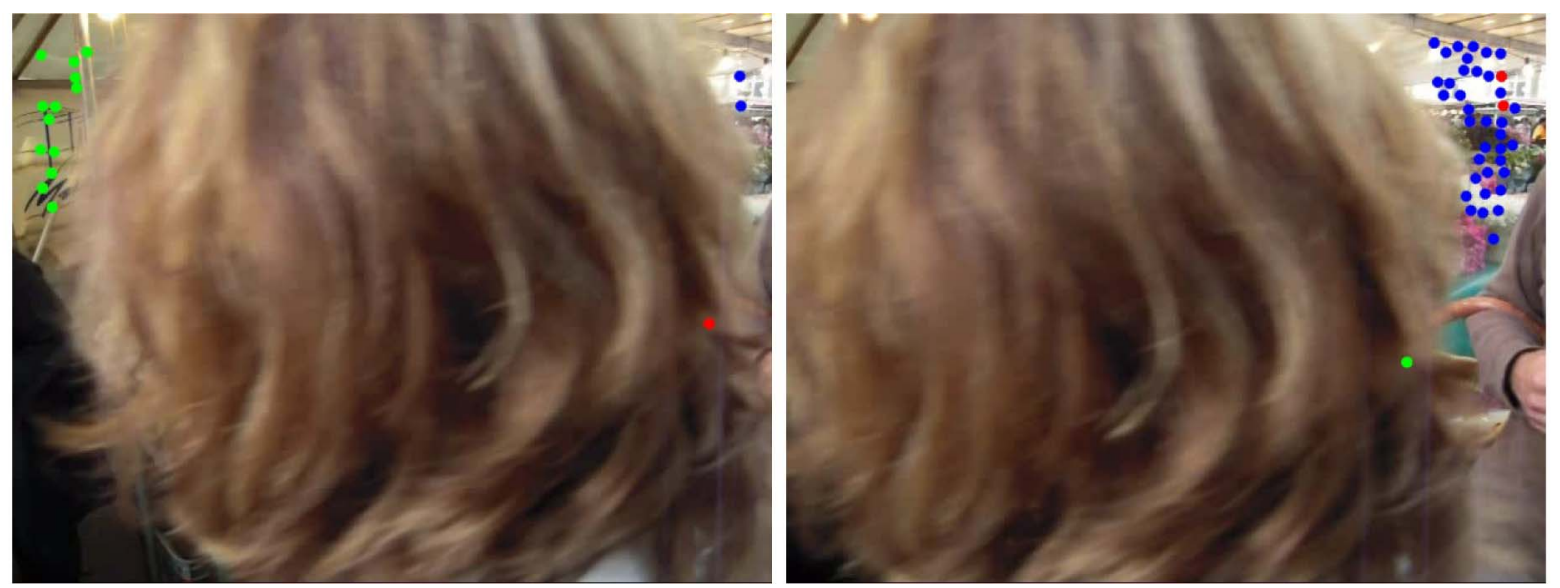

Fig. 6. From left to right are the consecutive frames. In this example, the appearing, existing and the missing features are shown in blue, red and green, respectively. The subspace [3] and the epipolar [4] methods fail at this example due to short feature trajectories. Our approach successfully stabilizes the video since the global optimization is solved.
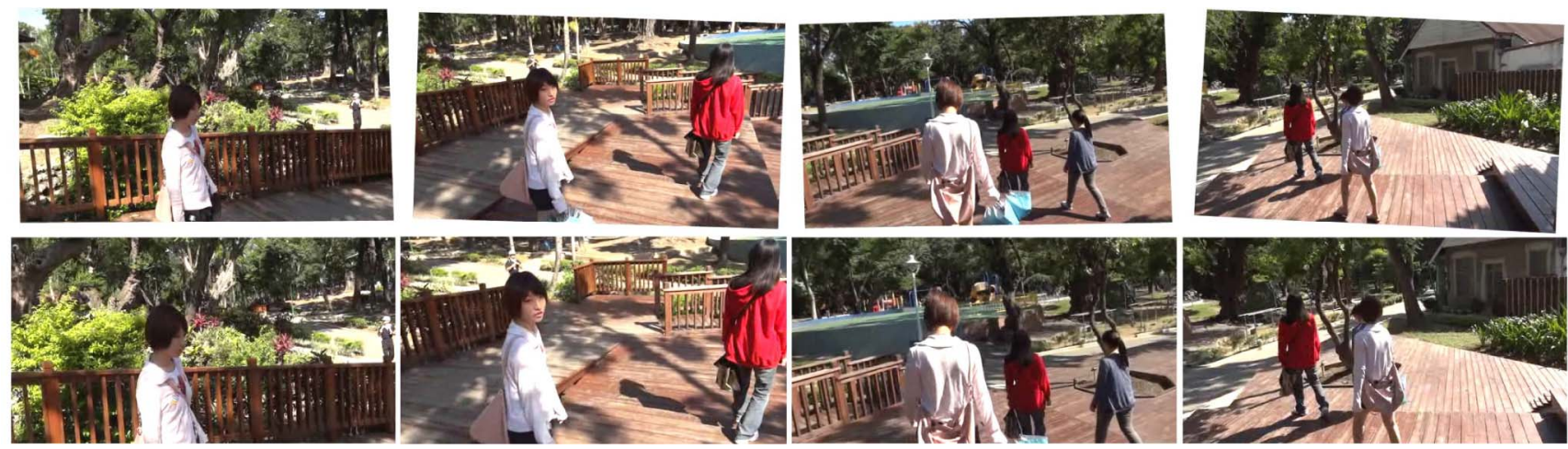

Fig. 7. We show the warped video frames (top row) and the cropped results (bottom row) that are determined by our system.

especially as the temporal stabilities are difficult to visualize and appreciate in still images.

Robustness. Our system can leverage very short feature trajectories because we solve a global optimization to achieve video stabilization. When sometimes the frame number of a trajectory $n-m+1$ may be smaller than the number of Bézier control points $d+1$, we represent that trajectory using the curve with degree $n-m$ in our implementation. Although our stabilizing result will be equal to the original video if all trajectories are two frames long, this extreme case rarely appears. Moreover, our reliability constraint allows some video frames to have even no features because constraining corner vertices to have smooth transitions ensures all unknowns being correlated when solving the optimization. Although the frames with no features may not be effectively stabilized in
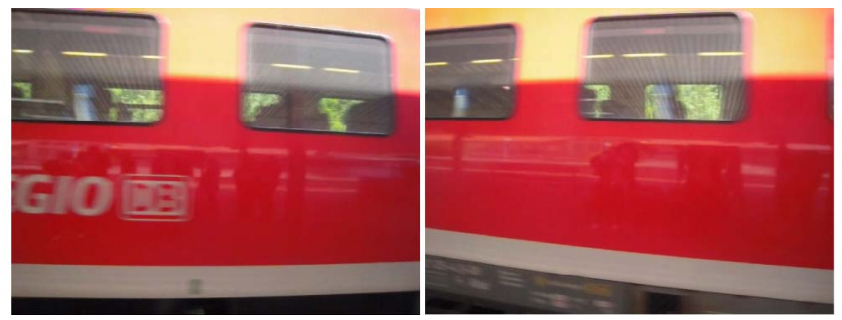

Fig. 8. Although our algorithm is robust to all challenging examples, the stabilization is not effective if there are no background features in some frames. this scenario, our system can produce the result for such a challenging example, which is much more stable than its original version (see Fig. 8 and our online supplemental video limitation.mp4).

Comparisons. We have compared our results with those stabilized by state-of-the-art techniques, including L1optimization [8], the subspace [3], and the epipolar [4] methods. The 3D stabilization [1] is not included because the latter two approaches [3], [4] can also handle parallax and have been demonstrated to be more robust. We show that our method can work well on videos that are shown in the paper of [3], [4], [8] in our online supplemental materials (CompToLiu.mp4, CompToGrundmann.mp4, and CompToGoldstein.mp4). Meanwhile, we focused on the selected challenging examples containing large parallax, strong occlusions and fast camera motions to demonstrate the effectiveness of our technique. Since the methods of [3], [4] fail at producing many results among these examples, we thus mainly compare our technique to [8] in our demo video Main.mp4. For those examples that the subspace and epipolar methods [3], [4] can stabilize, we show their results as well as ours in challenging.mp4.

The L1-optimization results we used for the comparison are from the YouTube Video Stabilizer, ${ }^{2}$ which is implemented based on Grundmann et al.'s technique [8]. This strategy strongly smoothes camera paths by solving

2. http://youtube.com/editor. 


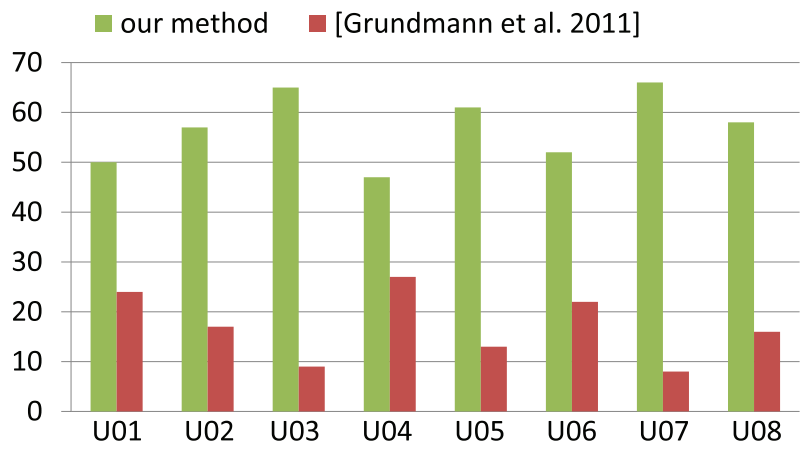

Fig. 9. The bar chart shows the participants' preferences among examples from U01 to U08.

inequality constraints. Although their system is robust to challenging examples, its $2 \mathrm{D}$ nature limits the ability to handle parallax, in which case global shaking potentially appears after stabilization, as demonstrated in the accompanying video. In contrast, we smooth feature trajectories in the spatial-temporal coordinate system to coherently transform features with different depth values and handle parallax well.

User study. Based on the selected challenging examples, we conducted a user study with 74 participants coming from diverse backgrounds and ages to evaluate our method. The participants were presented with an original video and two stabilizing results side by side, and they were asked to answer which result they prefer. During the study, the questions are presented in a random order to avoid bias. The results are automatically stabilized using [8] and our technique. The methods of [3], [4] were not included in the study because they fail to produce results for the examples due to the lack of long feature trajectories.

Fig. 9 shows the summary of the obtained results, which shows the significant preference of our method. Overall, it was favored over the method of [8] in 77 percent of 616 comparisons. In particular, the participants prefer our results that contain large parallax (see Fig. 9, U03.mp4, U07.mp4, and U08.mp4 in our online supplemental material) due to the less noticeable jittering artifacts. This obtained statistic is not unexpected, because our stabilization system can handle parallax well.

Limitations. Our system relies on the tracked background features to stabilize videos. Since even state-of-the-art feature trackers are not perfect, our stabilizing results are not always satisfactory. Some video frames potentially fall back to the shaky states due to excessive blur caused by extremely fast motions or lack of rigid background objects (see Fig. 8). In addition, the epipolar constraint may not remove the foreground features if their motions are slow. Our spatial rigidity preservation becomes insufficient in this scenario. Fortunately, the induced distortions are not visually noticeable because the relative positions of these foreground features to their neighboring background features change smoothly. Finally, as video frames are warped to achieve stabilization, our system may crop too much information if the given video is aggressively stabilized. We consider solving the mentioned problems in our future work.

\section{Conclusions AND Future Works}

We have introduced a robust, efficient, and streamable technique to video stabilization. Thanks to the spatial preservation of features in each video frame, our system successfully handles parallax without the reconstruction of a 3D scene. Although we achieve high robustness by solving a global optimization, our Bézier representation greatly reduces the computational cost when stabilizing a video. In addition, this strategy strongly stabilizes the video because the feature positions are linearly interpolated based on the optimized control points. Our technique matches or performs even better than the quality of state-of-the-art stabilizing systems while being robust to wide and diverse set of examples. We show the experimental results in our accompanying and the online supplemental videos to verify our technique.

Our streaming implementation achieves scalability and real-time performance when stabilizing long and highresolution videos, as long as KLT features are precomputed. Since real-time KLT implementations are currently available, ${ }^{3}$ we plan to embed this GPU-accelerated feature extraction into our system in the near future.

\section{ACKNOWLEDGMENTS}

The authors would like to thank the anonymous reviewers for their constructive comments. They are also grateful to Dr. Michael Adams for narrating the demo video and to PeiChih Wen for helping us with the experiments. This work was supported in part by the National Science Council (1012628-E-009-020-MY3, 100-2628-E-006-031-MY3, and 1002221-E-006-188-MY3), the NSF CNS-1205746, and the Portland State University Faculty Enhancement Grant.

\section{REFERENCES}

[1] F. Liu, M. Gleicher, H. Jin, and A. Agarwala, "Content-Preserving Warps for 3D Video Stabilization," ACM Trans. Graphics, vol. 28, pp. 44:1-44:9, 2009

[2] S. Liu, Y. Wang, L. Yuan, J. Bu, P. Tan, and J. Sun, "Video Stabilization with a Depth Camera," Proc. IEEE Conf. Computer Vision and Pattern Recognition (CVPR), 2012.

[3] F. Liu, M. Gleicher, J. Wang, H. Jin, and A. Agarwala, "Subspace Video Stabilization," ACM Trans. Graphics, vol. 30, no. 1, pp. 1-10, 2011.

[4] A. Goldstein and R. Fattal, "Video Stabilization Using Epipolar Geometry," ACM Trans. Graphics, vol. 31, pp. 1-10, 2012.

[5] W.-Q. Yan and M.S. Kankanhalli, "Detection and Removal of Lighting \& Shaking Artifacts in Home Videos," Proc. 10th ACM Int'l Conf. Multimedia, pp. 107-116, 2002.

[6] S. Wu and Z. Ren, "Video Stabilization by Multi-Trajectory Mapping and Smoothing," Proc. Fifth Int'l Conf. Information, Comm. and Signal Processing, pp. 542-545, 2005.

[7] M. Long, L. Xinggang, X. Lidong, and F. Fangwen, "Video Stabilizing System for Digital Camera," Proc. Int'l Conf. Signal Processing, pp. 1119-1122, 2004.

[8] M. Grundmann, V. Kwatra, and I. Essa, "Auto-Directed Video Stabilization with Robust 11 Optimal Camera Paths," Proc. IEEE Conf. Computer Vision and Pattern Recognition (CVPR), 2011.

[9] C. Morimoto and R. Chellappa, "Evaluation of Image Stabilization Algorithms," Proc. IEEE Int'l Conf. Acoustics, Speech and Signal Processing, pp. 2789-2792, 1998.

[10] Y. Matsushita, E. Ofek, W. Ge, X. Tang, and H.-Y. Shum, "FullFrame Video Stabilization with Motion Inpainting," IEEE Trans. Pattern Analysis and Machine Intelligence, vol. 28, no. 7, pp. 11501163, July 2006.

3. http://cs.unc.edu/ ssinha/Research/GPU_KLT/. 
[11] J. Yang, D. Schonfeld, C. Chen, and M.A. Mohamed, "Online Video Stabilization Based on Particle Filters," Proc. Int'l Conf. Image Processing (ICIP), pp. 1545-1548, 2006.

[12] B.-Y. Chen, K.-Y. Lee, J.-S. Lin, and W.-T. Huang, “Capturing Intention-Based Full-Frame Video Stabilization," Computer Graphics Forum, vol. 27, pp. 1805 -1814, 2008.

[13] M. Gleicher and F. Liu, "Re-Cinematography: Improving the Camerawork of Casual Video," ACM Trans. Multimedia Computing Comm. and Applications, vol. 5, no. 1, pp. 1-28, 2008.

[14] K.-Y. Lee, Y.-Y. Chuang, B.-Y. Chen, and M. Ouhyoung, "Video Stabilization Using Robust Feature Trajectories," Proc. IEEE 12th Int'l Conf. Computer Vision (ICCV), pp. 1397-1404, 2009.

[15] A. Fitzgibbon, Y. Wexler, and A. Zisserman, "Image-Based Rendering Using Image-Based Priors," Int'l J. Computer Vision, vol. 63, no. 2, pp. 141-151, 2005.

[16] P. Bhat, C.L. Zitnick, N. Snavely, A. Agarwala, M. Agrawala, M. Cohen, B. Curless, and S.B. Kang, "Using Photographs to Enhance Videos of a Static Scene," Proc. Eurographics Workshop Rendering, pp. 327-338, 2007.

[17] G. Zhang, W. Hua, X. Qin, Y. Shao, and H. Bao, "Video Stabilization Based on a 3D Perspective Camera Model," The Visual Computer, vol. 25, no. 11, pp. 997-1008, 2009.

[18] Z. Huang, F. He, X. Cai, Y. Chen, and X. Chen, "A 2D-3D Hybrid Approach to Video Stabilization," Proc. IEEE Int'l Conf. CS-Aided Design and CS Graphics, pp. 146-150, 2011.

[19] S. Baker, E.P. Bennett, S.B. Kang, and R. Szeliski, "Removing Rolling Shutter Wobble," Proc. IEEE Conf. Computer Vision and Pattern Recognition (CVPR), pp. 2392-2399, 2010.

[20] E. Ringaby and P.-E. Forssén, "Efficient Video Rectification and Stabilisation for Cell-Phones," Int'l J. Computer Vision, vol. 96, pp. 335-352, 2012.

[21] M. Grundmann, V. Kwatra, D. Castro, and I. Essa, "CalibrationFree Rolling Shutter Removal," Proc. Int'l Conf. Computational Photography, 2012.

[22] J. Shi and C. Tomasi, "Good Features to Track," Proc. IEEE CS Conf. Computer Vision and Pattern Recognition (CVPR), pp. 593-600, 1994.

[23] R.I. Hartley and A. Zisserman, Multiple View Geometry in Computer Vision. Cambridge Univ. Press, 2000.

[24] O. Sorkine and M. Alexa, "As-Rigid-as-Possible Surface Modeling," Proc. EUROGRAPHICS/ACM SIGGRAPH Symp. Geometry Processing, pp. 109-116, 2007,

[25] "Voodoo Camera Tracker: A Tool for the Integration of Virtual and Real Scenes,"http://www.digilab.uni-hannover.de/docs/ manual.html, 2013.

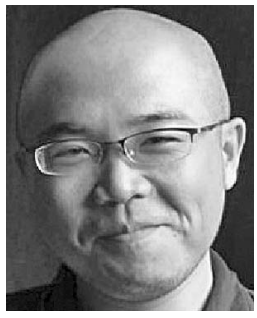

Yu-Shuen Wang received the BSc and $\mathrm{PhD}$ degrees from the Department of Computer Science and Information Engineering, National Cheng-Kung University, in 2004 and 2010, respectively. He is currently an assistant professor of the Department of Computer Science at National Chiao-Tung University (http://people.cs.nctu.edu.tw/ yushuen/). He leads the Computer Graphics and Visualization Lab at the Institute of Multimedia Engineering. His research interests include computer graphics, computational photography, and visualization.

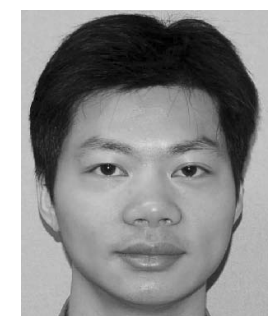

Feng Liu received the BS and MS degrees in computer science from Zhejiang University, in 2001 and 2004, respectively, and the MS and $\mathrm{PhD}$ degree in computer science from the University of Wisconsin, Madison, in 2006 and 2010 , respectively. $\mathrm{He}$ is an assistant professor in the Department of Computer Science at Portland State University. His research interests include the areas of computer graphics, vision, and multimedia. He is a 2012 Intel Early Career Faculty Honor Program awardee. He is a member of the IEEE and the IEEE Computer Society.

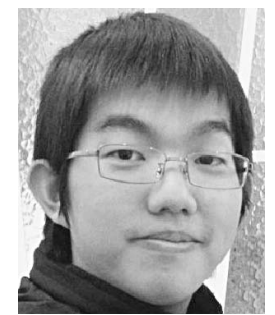

Pu-Sheng Hsu received the MS degree from the Department of Computer Science and Information Engineering, National Cheng-Kung University, Taiwan, in 2011. Now, he is working in High Tech Computer Corporation. His research interests include computer graphics and video processing.

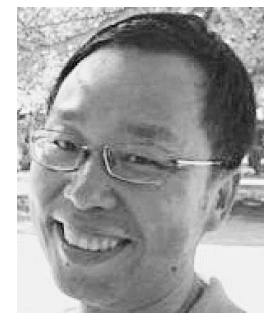

Tong-Yee Lee received the $\mathrm{PhD}$ degree in computer engineering from Washington State University, Pullman, in May 1995. He is currently a distinguished professor in the Department of Computer Science and Information Engineering, National Cheng-Kung University, Tainan, Taiwan, R.O.C. He leads the Computer Graphics Group, Visual System Laboratory, National Cheng-Kung University (http://graphics.csie. ncku.edu.tw/). His current research interests include computer graphics, nonphotorealistic rendering, medical visualization, virtual reality, and media resizing. He also serves on the editorial boards of the IEEE Transactions on Information Technology in Biomedicine, the Visual Computer and the Computers and Graphics Journal. He served as a member of the international program committees of several conferences including the IEEE Visualization, the Pacific Graphics, the IEEE Pacific Visualization Symposium, the IEEE Virtual Reality, the IEEE-EMBS International Conference on Information Technology and Applications in Biomedicine, and the International Conference on Artificial Reality and Telexistence. He is a member of the ACM and a senior member of the IEEE.

For more information on this or any other computing topic please visit our Digital Library at www.computer.org/publications/dlib. 